\title{
Optical sensing of chlorophyll(in) with dual-spectrum Si LEDs in SOI-CMOS technology
}

\author{
Satadal Dutta, Peter G. Steeneken, and Gerard J. Verbiest
}

\begin{abstract}
Small and low-cost chlorophyll sensors are popular in agricultural sector and food-quality control. Combining such sensors with silicon CMOS electronics is challenged by the absence of silicon-integrated light-sources. We experimentally achieve optical absorption sensing of chlorophyll based pigments with silicon (Si) micro light-emitting diodes (LED) as light-source, fabricated in a standard SOI-CMOS technology. By driving a Si LED in both forward and avalanche modes of operation, we steer its electroluminescent spectrum between visible $(400-900 \mathrm{~nm})$ and near-infrared $(\sim 1120 \mathrm{~nm})$. For detection of chlorophyll in solution phase, the dual-spectrum light from the LED propagates vertically through glycerol micro-droplets containing sodium copper chlorophyllin at varying relative concentrations. The transmitted light is detected via an off-chip Si photodiode. The visible to near-infrared color ratio (COR) of the photocurrent yields the effective absorption coefficient. We introduce the LED-specific molar absorption coefficient as a metric to compute the absolute pigment concentration $\left(\sim 0.019 \pm 0.006 \mathrm{~mol} \mathrm{~L}^{-1}\right)$ and validate the results by measurements with a hybrid spectrophotometer. With the same sensor, we also show non-invasive monitoring of chlorophyll in plant leaves. COR sensitivities of $\sim 3.9 \times 10^{4} \mathrm{~mol}^{-1} \mathrm{~L}$ and $\sim 5.3 \times 10^{4} \mathrm{~mol}^{-1} \mathrm{~L}$ are obtained for two leaf species, where light from the LED propagates diffusely through the thickness of the leaf prior to detection by the photodiode. Our work demonstrates the feasibility of realizing fully CMOS-integrated optical sensors for biochemical analyses in food sector and plant/human health.
\end{abstract}

Index Terms-Silicon, Avalanche breakdown, CMOS, electroluminescence, optical sensor, light-emitting diode, chlorophyll.

\section{INTRODUCTION}

Chlorophyll (Chl) is an essential pigment for photosynthesis and a key indicator of health of plants [1]. Moreover, chemically stable derivatives of Chl, e.g. chlorophyllins, are key ingredients in a plethora of dietary and medicinal supplements [2]. Sensors to monitor both natural Chl content in leaves and chlorophyllin in food supplements are, thus, widely used in the agricultural sector and for food-quality control [3]-[5]. Optical sensing is highly suitable to detect $\mathrm{Chl}$ and its derivatives. This is due to the strong interaction of these pigments with ultraviolet and visible light [6]. While leaf transmittance measurement is popular to monitor $\mathrm{Chl}$ non-invasively [7], colorimetric detection of pigments in compact microfluidic systems [8] (and references therein) is

An earlier version of this paper was presented at the IEEE Sensors Conference 2020 and was published in its Proceedings: https://ieeexplore.ieee.org/document/9278700. The work was carried out under the Plantenna research program funded by the 4TU Federation of the Netherlands.

S. Dutta, G.J. Verbiest and P.G. Steeneken are with the Department of Precision and Microsystems Engineering, Delft University of Technology, 2628CD Delft, The Netherlands, e-mail: (s.dutta-1@tudelft.nl; g.j.verbiest@tudelft.nl; p.g.steeneken@tudelft.nl). becoming popular to ensure fast detection, simple operation and small volume of sample. Advances in semiconductor technology have led to a variety of hybrid light-sources in the same spectral window, e.g. lasers and quasi-monochromatic light-emitting diodes (LEDs), that are employed in the state-of-the-art optical sensors [9] [10]. However, these lightsources are incompatible with silicon ( $\mathrm{Si}$ ) CMOS technology, which is the workhorse for advanced electronic read-out and data processing modules for sensors [11]. This hinders device integration and makes these sensors relatively expensive and bulky.

Silicon photonics is emerging as a key technology for the development of CMOS-integrated optical devices for applications in bio-chemical sensing and data communication links [12]-[19]. Interestingly, Si p-n junction diodes exhibit broad-spectrum electroluminescence (EL) at wavelengths $(\lambda)$ near $1120 \mathrm{~nm}$ in forward mode (FM) and at $\lambda$ in the range of 400-900 $\mathrm{nm}$ in avalanche mode (AM) operation. Although this EL occurs at a low quantum efficiency $\left(\sim 10^{-3}-10^{-5}\right)$ [20]-[26] due to the indirect bandgap of $\mathrm{Si}$, for many applications the advantages of CMOS integration 

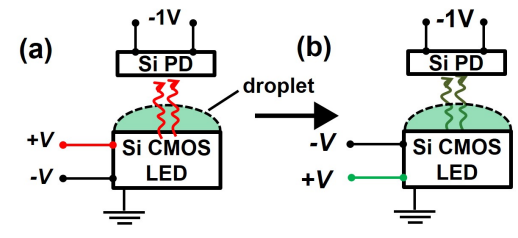

Forward $\quad \lambda: 1000 \mathrm{~nm}-$
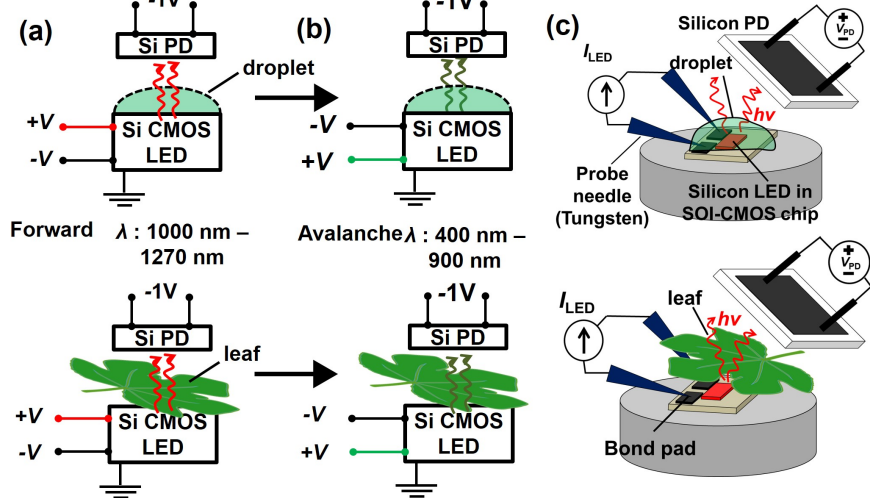

Fig. 1. Schematic block diagram illustrating the optical sensing method. Optical coupling from the Si LED on the CMOS chip to an externally mounted Si PD (reverse biased at $1 \mathrm{~V}$ ) is measured in presence of a pigmented glycerol droplet (top panel) or a leaf (bottom panel) on top of the LED. The LED is operated in (a) forward mode (FM) and (b) avalanche mode (AM). The pigment (NaCuChl) absorbs light in the range $400<\lambda<800 \mathrm{~nm}$. (c) Schematic of the measurement setup (not to scale). The on-chip LED is placed on a vacuum chuck and is driven in constant current mode, where electrical probing is done via aluminium bond-pads and tungsten needles. The wavy arrows indicate light propagation.

of the LED outweigh the drawback of low efficiency. Recent advancements [27]-[29] have successfully highlighted the $\mathrm{Si}$ LED as a promising candidate for monolithically integrated optical interconnects due to the high responsivity of $\mathrm{Si}$ photodiodes (PDs) for wavelengths $(\lambda)<1000 \mathrm{~nm}$. The ability to electrically switch between visible (VIS) and nearinfrared (NIR) emission from a single Si LED eliminates the need for any process modification or device replacement in an optical sensor. In contrast, wavelength tuning in LEDs made of III-V semiconductors require large stoichiometric changes in material composition [30]. The $400-1300 \mathrm{~nm}$ spectral range is highly suitable for sensing photosynthetic pigments [6], leaf-water status [33], [34], colored contaminants in water [35]-[37] and blood oxygen levels [38].

In this work, we show how $\mathrm{Si}$ LEDs fabricated in a standard SOI-CMOS technology are viable light-sources for use in optical absorption sensing systems, by driving a single LED in both FM and AM operation. The emitted light propagates vertically through a pigmented micro-droplet placed on the surface followed by detection by a discrete $\mathrm{Si}$ PD mounted externally above the chip (see Fig. 1(a), (b)). We define the color ratio (COR) as ratio of PD photocurrent during AM LED operation to that during FM LED operation. From the COR and the mean height of the droplet (optical path length), we obtain the effective absorption coefficient $(\alpha)$ of the pigmented solution specific to our broadband $\mathrm{Si}$ LED. In our recent conference proceedings paper [31], we demonstrated this sensing principle using Carmine pigment. In this paper, we extend our work by sensing Chl-based pigments, namely sodium copper chlorophyllin $(\mathrm{NaCuChl})$ in solution phase and chlorophyll $(\mathrm{Chl})$ in intact plant-leaves.

The rest of the manuscript is organised as follows. Section (a)
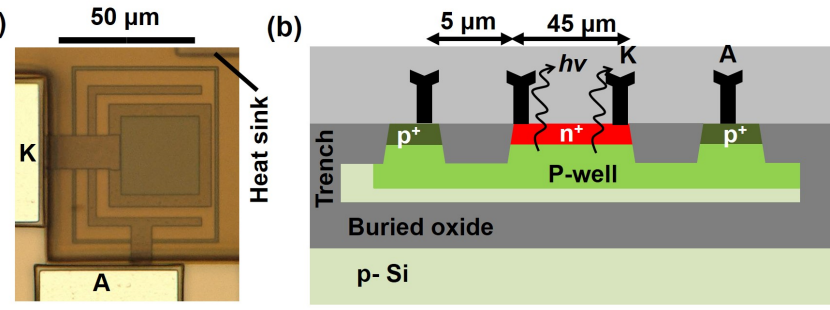

Fig. 2. (a) Top-view micrograph and (b) device cross-section of the $n+p$ junction LED in a SOI-CMOS technology from NXP Semiconductors [32]. Symbols $A$ and $K$ indicate the anode and cathode terminating in Al bond pads. The LED has a junction area of $45 \times 45 \mu \mathrm{m}^{2}$, and a reverse breakdown voltage of $\sim 17 \mathrm{~V}$.

II describes the experimental device and the method. Sections III-B and III-C present the measurement results and data analyses for sensing with droplets (containing $\mathrm{NaCuChl}$ in solution) and with intact leaves (containing naturally occurring Chl.), respectively. Section IV briefly outlines the merits, applicability and future scope of the sensor. Section V summarizes our work.

\section{EXPERIMENTAL SET-UP AND METHOD}

Fig. 1(c) shows a schematic diagram of the set-up. The on-chip Si LED is placed on a vacuum chuck. The bond-pads are electrically probed by Tungsten needles connected to EverBeing EB-050 micropositioners in a probe station. A BPW34 Si PD from Vishay semiconductors is mounted above the chip at a center-height $\sim 5 \mathrm{~mm}$ tilted at $\sim 45$ degrees via a micropositioner. The LED is driven in a constant current (sweep) mode and the PD is driven at a fixed reverse bias of $1 \mathrm{~V}$ using a Keysight B2912A precision source and measurement unit (SMU). During measurement, either a droplet with $\mathrm{NaCuChl}$. or a leaf is placed on top of the Si LED.

Figs. 2(a) and (b) show, respectively, the optical top-view micrograph (layout), and the schematic device cross-section of the test LED, designed in a standard $130 \mathrm{~nm}$ siliconon-insulator (SOI) CMOS technology [32], from NXP Semiconductors. The LED is a vertical $\mathrm{n}^{+} \mathrm{p}$ junction diode with a junction depth of $\sim 0.25 \mu \mathrm{m}$, an active junction area of $45 \times 45 \mu \mathrm{m}^{2}$, and an avalanche breakdown voltage of $\sim 17 \mathrm{~V}$ [27]. Vertical poly-Si columns are placed near the p-n junction, which connect the SOI layer to the Si substrate. They serve as heat sinks to reduce the thermal resistance significantly.

In a first experiment, we test the sensing principle using droplets containing $\mathrm{NaCuChl}$. A commercially available solution $(\sim 10 \mathrm{mM})$ of $\mathrm{NaCuChl}$ (E141), from PipingRock Health Products, LLC. is used as a target. We choose glycerol as the solvent for the droplets. This choice is motivated by its negligible volatility at $300 \mathrm{~K}$, optical transparency for $\lambda$ in the range 400-1200 nm, and chemical inertness to the $\mathrm{SiO}_{2}$ layer on the chip surface. We denote the $\mathrm{NaCuChl}$. concentration of the target solution as $c_{\text {ref. }}$. This solution is diluted in appropriate volumes of excess glycerol to yield solution specimens of the following relative concentrations $\left(c / c_{\text {ref }}\right)$ of 


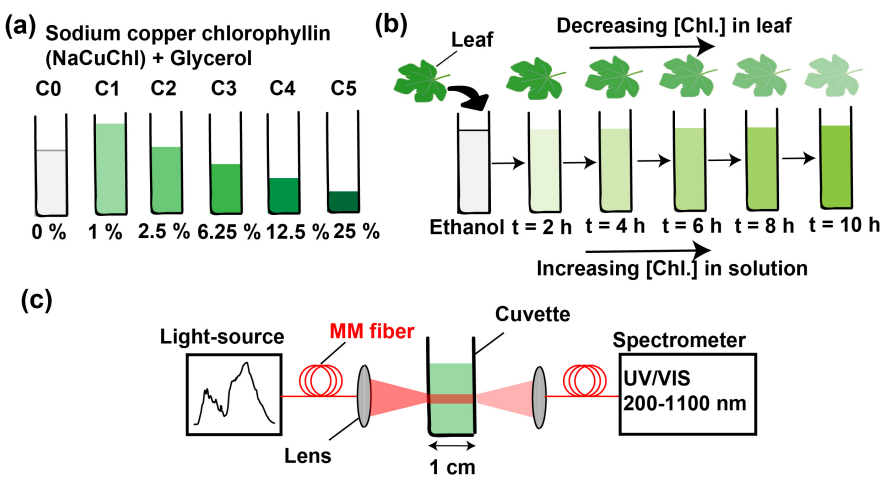

Fig. 3. (a) Solution specimens of sodium copper chlorophyllin (NaCuChl) diluted in laboratory-grade glycerol with indicated concentrations (\% by volume) relative to a reference commercial solution $(\sim 10 \mathrm{mM})$ of $\mathrm{NaCuChl}(\mathrm{E} 141)$ in glycerol and water. C0 represents only glycerol. (b) Method for Chl. monitoring in leaves. To emulate chlorophyll deficiency, the test leaf is treated with $12 \mathrm{~mL}$ ethanol at $300 \mathrm{~K}$ in a graduated vial. At every 2 hour interval, optical transmission through the leaf is measured with the sensor and the absorbance of the ethanol extract is measured with the spectrophotometer set-up as illustrated in (c). A fiber-coupled AvaLight-DH-S-BAL light-source (Deuterium and halogen) shines a collimated beam through the solution (C1) in a UVcuvette with $1 \mathrm{~cm}$ optical path. The transmitted beam is fed to an AvaSpec-ULS2048CL-EVO spectrometer $(200-1100 \mathrm{~nm})$ via a multimode $400 \mu \mathrm{m}$ core optical fiber.

$\mathrm{C} 1=1.0 \%, \mathrm{C} 2=2.5 \%, \mathrm{C} 3=6.25 \%, \mathrm{C} 4=12.5 \%$, and $\mathrm{C} 5=25.0 \%$, as shown in Fig. 3(a). We label our solvent as C0 $(c=0)$. The PD photocurrent $\Delta I_{\mathrm{PD}}$ ( $\Delta$ represents that the dark current has been subtracted) is measured in air and in presence of a micro-droplet, which is transferred from each solution specimen with a hydrophilic tip of a $\sim 100 \mu \mathrm{m}$ core silica fiber. The droplets-on-chip have lateral diameters $>500 \mu \mathrm{m}$, and hence covers the on-chip LED entirely (Fig. 1(c)), as was shown in our earlier work [31]. The same droplet is used to measure $\Delta I_{\mathrm{PD}}$ by driving the same LED in $\mathrm{AM}$ and FM operation at bias currents $I_{\mathrm{LED}}=1-6 \mathrm{~mA}$.

In the second experiment, we collect fresh green leaf specimens, one each from Hamamelis virginiana L. and Capsicum annuum L. plants. The leaves are then washed with demi water and wiped dry to remove dust and impurities from the surface. The PD photocurrent is then measured with the setup described above, with the adaxial surface of the fresh leaf facing the on-chip LED (Fig. 1(c)). Each leaf is inserted into a vial containing $12 \mathrm{~mL}$ of ethanol at $300 \mathrm{~K}$. The ethanol treatment emulates $\mathrm{Chl}$ stress in the leaf as the photosynthetic pigments dissolve in ethanol. After time $t=2,4,6,8$, and 10 hours, the leaf is withdrawn from its vial (Fig. 3(b)) and the corresponding PD photocurrent is measured. At each timestamp, the ethanolic extract is also sampled to measure its spectral absorbance with a hybrid spectrophotometer set-up illustrated in Fig. 3(c). This set-up consists of a fiber-coupled AvaLight-DH-S-BAL light source (Deuterium plus halogen) that shines a collimated beam through a UV-cuvette containing the test solution with $1 \mathrm{~cm}$ optical path. The transmitted beam is fed to an AvaSpec-ULS2048CL-EVO spectrometer (200$1100 \mathrm{~nm}$ ) via a multi-mode $400 \mu \mathrm{m}$ core optical fiber.

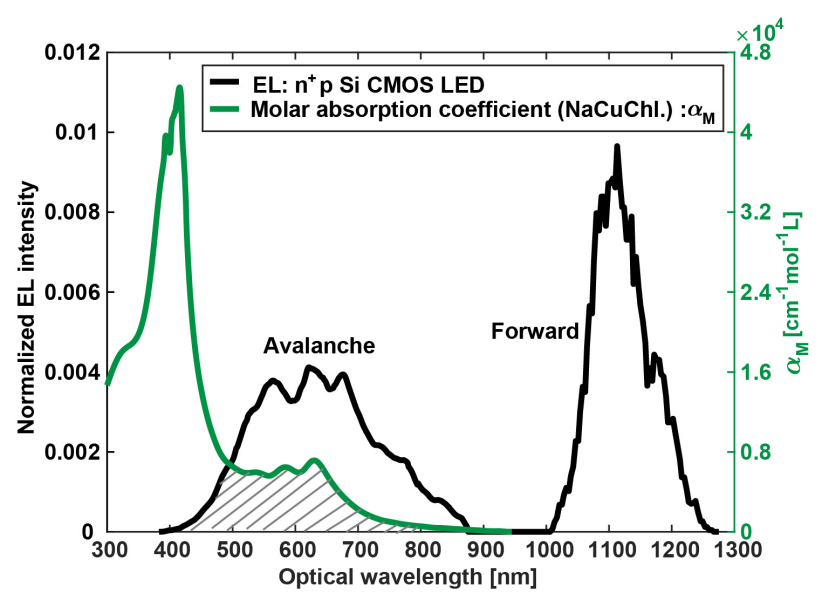

Fig. 4. Avalanche-mode (400-900 nm) and forward-mode (1000$1270 \mathrm{~nm}$ ) normalized electroluminescent intensity spectra of the Si LED (in black) measured with a ADC-1000-USB and AvaSpec-UV/VIS/NIR spectrometers respectively from Avantes B.V. The measured molar absorption coefficient spectrum (in green) of $\mathrm{NaCuChl}$ in glycerol is measured with the hybrid spectrophotometer set-up shown in Fig. 3(c), and calibrating the value at $\lambda=626 \mathrm{~nm}$ from [2].

\section{Results AND ANALYSis}

\section{A. LED EL-spectra and NaCuChl molar absorption coefficient}

Light emission from forward-biased $\mathrm{Si}$ diodes at $\lambda$ in the range 1000-1270 $\mathrm{nm}$, results from indirect (phonon-assisted) recombination of electron-hole pairs near the band-edge [20]. When the diode is reverse biased above the avalanche breakdown voltage, the high electric field in the depletion region accelerates the charge carriers to energies exceeding the bandgap of $1.12 \mathrm{eV}$. This leads to high-energy inter-band electronic transitions, resulting in a broad-spectrum EL peaking in the 600-700 nm wavelength window [22], [23], [25]. Fig. 4 shows the normalized EL spectra of the $n^{+}$p junction LED [27], [31] measured in avalanche-mode (AM) and forward-mode (FM) operation, with a UV/VIS (ADC-1000-USB) and AvaSpecNIR spectrometer from Avantes B.V., respectively. The power normalized EL spectral irradiance $\varepsilon(\lambda)\left[\mathrm{nm}^{-1}\right]$ is defined by:

$$
\varepsilon(\lambda)=\frac{E(\lambda)}{\int_{\lambda} E(\lambda) \cdot \mathrm{d} \lambda},
$$

where $E(\lambda)$ is the measured EL intensity, and integration limits are from $\lambda=400 \mathrm{~nm}$ till $\lambda=900 \mathrm{~nm}$. The AM EL spectrum has a significant overlap with the absorption spectrum of $\mathrm{NaCuChl}$, whereas the same chemical absorbs negligibly in the FM EL spectrum of the Si LED. Fig. 4 also shows the molar absorption coefficient $\alpha_{\mathrm{M}}(\lambda)$ of our $\mathrm{NaCuChl}$ solution in glycerol. The specimen $\mathrm{C} 1\left(c_{1} / c_{\text {ref }}=0.01\right)$ is used for this purpose and the absorbance $A(\lambda)$ is measured with the spectrophotometer set-up. The absorption coefficient of the specimen $\mathrm{C} 1, \alpha_{\mathrm{C} 1}(\lambda)\left[\mathrm{cm}^{-1}\right]$ is calculated from BeerLambert's law as:

$$
\alpha_{\mathrm{C} 1}(\lambda)=-\frac{\ln \left(1-A_{\mathrm{C} 1}(\lambda)\right)}{L_{\mathrm{opt}}},
$$

where $L_{\mathrm{opt}}=1 \mathrm{~cm}$ is the optical path length of the light beam in the cuvette (see Fig. 3(c)). Subsequently, $\alpha_{M}(\lambda)$ 


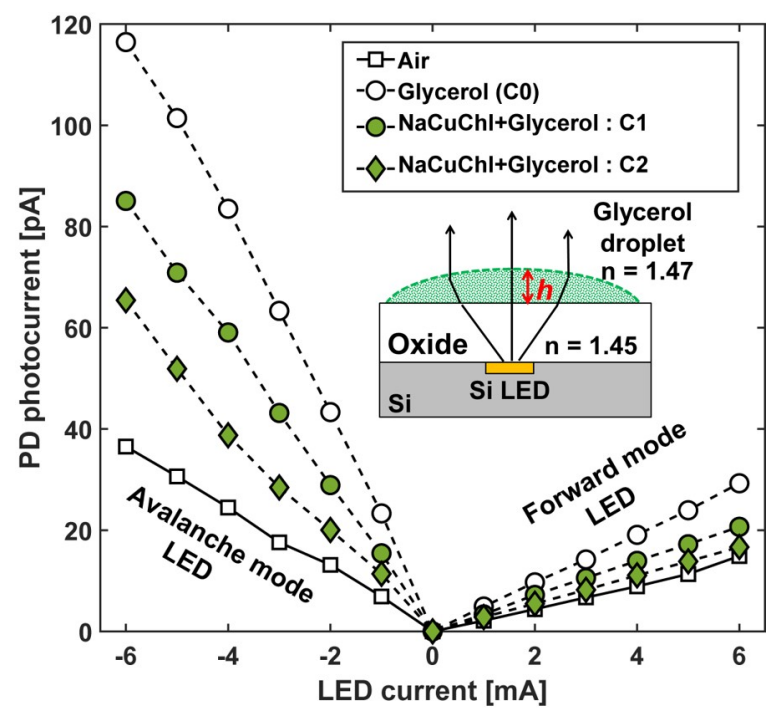

Fig. 5. Photocurrent (at reverse bias of $1 \mathrm{~V}$ ) in the Si PD versus LED current in avalanche (-X axis) and forward $(+X$ axis) modes of operation in air (squares), in presence of glycerol droplets from specimens $\mathrm{CO}$ (white circles), C1 (green circles) and C2 (green diamonds). (Inset) : Schematic cross-section showing the light-rays being focused by the plano-convex micro-lens formed by the droplet.

$\left[\mathrm{cm}^{-1} \mathrm{~mol}^{-1} \mathrm{~L}\right]$ is estimated as :

$$
\alpha_{\mathrm{M}}(\lambda)=\left(\frac{c_{\mathrm{ref}}}{c_{1}}\right) \cdot\left(\frac{\alpha_{\mathrm{C} 1}(\lambda)}{\alpha_{\mathrm{C} 1}(626 \mathrm{~nm})}\right) \cdot 7192
$$

where we have used the literature reported molar absorption coefficient of $\mathrm{NaCuChl}$ as $7192 \mathrm{~cm}^{-1} \mathrm{~mol}^{-1} \mathrm{~L}$ at $\lambda=626 \mathrm{~nm}$ (Q-band) [2] as a calibration point.

In order to use the Si LED as the light source for optical transmission measurements, we first compute the LED-specific molar absorption coefficient $\alpha_{\mathrm{M}}^{\mathrm{LED}}\left[\mathrm{cm}^{-1} \mathrm{~mol}^{-1} \mathrm{~L}\right]$, and is expressed as:

$$
\alpha_{\mathrm{M}}^{\mathrm{LED}}=\int_{\lambda} \alpha_{\mathrm{M}}(\lambda) \cdot \varepsilon(\lambda) \cdot \mathrm{d} \lambda,
$$

where the limits of integration are the same as in eq. (1). We obtain $\alpha_{\mathrm{M}}^{\mathrm{LED}}=4782.7 \mathrm{~cm}^{-1} \mathrm{~mol}^{-1} \mathrm{~L}$. Note that $\alpha_{\mathrm{M}}^{\mathrm{LED}}$ is a quantitative way to express the overlap between the $\varepsilon(\lambda)$ and $\alpha_{\mathrm{M}}(\lambda)$ (indicated by the shaded region in Fig. 4).

\section{B. Sodium copper chlorophyllin in glycerol-droplet}

Fig. 5 shows the measured PD photocurrent (at a reverse bias of $1 \mathrm{~V}$ ) which varies linearly with $I_{\mathrm{LED}}$ in both AM and FM LED operation, showing a linear relationship between optical power and $I_{\mathrm{LED}}$. We observe that $\Delta I_{\mathrm{PD}}^{\mathrm{AM}}>\Delta I_{\mathrm{PD}}^{\mathrm{FM}}$ at any given $I_{\mathrm{LED}}$. This is primarily due to the higher PD quantum efficiency for light emitted in AM operation [27]. Further, we observe that both $\Delta I_{\mathrm{PD}}^{\mathrm{AM}}$ and $\Delta I_{\mathrm{PD}}^{\mathrm{FM}}$ are, respectively, a factor of $\sim 3.2$ and $\sim 2.0$ higher in presence of the glycerol droplet $(\mathrm{C} 0)$ as compared to transmission in air. This is explained by two factors. Firstly, there is a good refractive index matching between glycerol $(\mathrm{n} \approx 1.47)$ and

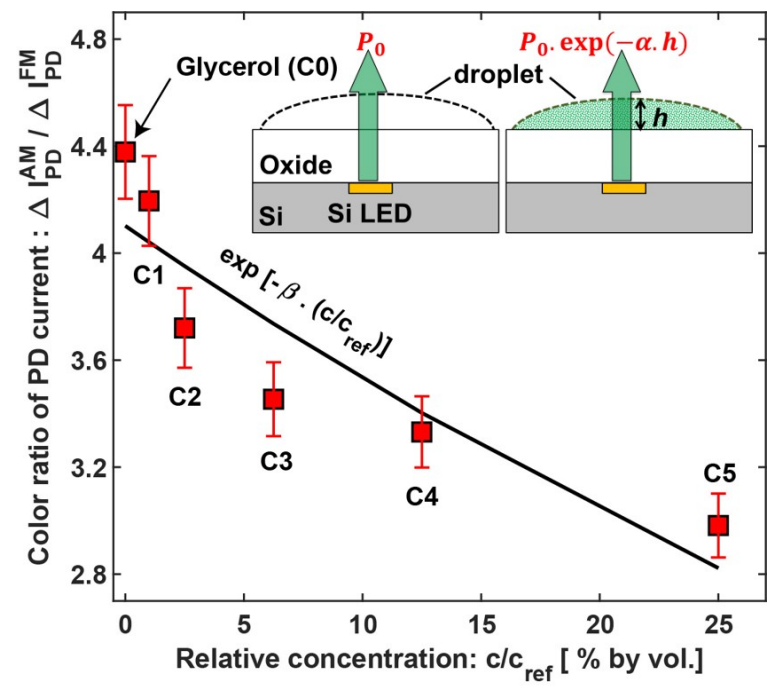

Fig. 6. Color ratio (AM to FM operation of the Si CMOS LED) of the PD photocurrent when light transmission occurs through the microdroplets sampled from solution specimens $\mathrm{CO}$ to $\mathrm{C} 5$. The values for COR are averaged over $\boldsymbol{I}_{\mathbf{L E D}}=1-6 \mathrm{~mA}$. COR decreases exponentially with increasing $c$ (black solid line). The fitted attenuation constant $\boldsymbol{\beta}=\left(\boldsymbol{\alpha}_{\mathrm{ref}}^{\mathrm{LED}} \cdot \boldsymbol{h}\right)$ is a function of the effective absorption coefficient of the reference $\mathrm{NaCuChl}$. solution, and the droplet height $\boldsymbol{h}$ (see inset).

$\mathrm{SiO}_{2}(\mathrm{n} \approx 1.45)$ over the droplet-covered die area. Secondly, the droplet acts as a plano-convex lens leading to a gain in the optical extraction efficiency (see inset of Fig. 5). A mismatch in the optical extraction efficiency between AM and FM operation is likely due to possible electrostatic effects on the shape or on the refractive index [39] of the glycerol droplet at widely different values of LED bias voltages : $\sim 19 \mathrm{~V}$ for $\mathrm{AM}$ and $\sim 1 \mathrm{~V}$ for FM LED. Since the pigment absorption window overlaps only with the AM EL spectrum, we can express the photocurrent in presence of the droplet for FM and AM LED operation, respectively as

$$
\Delta I_{\mathrm{PD}(\mathrm{Gly})}^{\mathrm{FM}}\left(c_{i}\right)=\eta_{\mathrm{LED}}^{\mathrm{FM}} \cdot \eta_{\mathrm{PD}}^{\mathrm{FM}} \cdot \eta_{\mathrm{Gly}}^{\mathrm{FM}} \cdot I_{\mathrm{LED}}
$$

$$
\Delta I_{\mathrm{PD}(\mathrm{Gly})}^{\mathrm{AM}}\left(c_{i}\right)=\eta_{\mathrm{LED}}^{\mathrm{AM}} \cdot \eta_{\mathrm{PD}}^{\mathrm{AM}} \cdot \eta_{\mathrm{Gly}}^{\mathrm{AM}} \cdot I_{\mathrm{LED}} \cdot \exp \left[-\alpha\left(c_{i}\right) \cdot h\right]
$$

where $h$ is the droplet height, and $c_{i}$ represents the concentration of the $i$ th solution $(i=0,1,2,3,4,5)$. Here $\eta_{\mathrm{LED}}$ and $\eta_{\mathrm{PD}}$ represent the external quantum efficiencies of light-emission from the LED and light-detection by the $\mathrm{PD}$, respectively. $\eta_{\text {Gly }}$ is the light-extraction efficiency from the chip through the droplet. Considering a measurement error of $\pm 2 \mathrm{pA}$ in the photocurrent, we observe in Fig. 5 that for droplets containing $\mathrm{NaCuChl}$., absorption of visible light by the pigment leads to a decrease in $\Delta I_{\mathrm{PD}}^{\mathrm{AM}}$, as compared to transmission via the pure glycerol droplet. Small discrepancies in $\Delta I_{\mathrm{PD}}^{\mathrm{FM}}$ among droplet specimens are likely due to uncertainties in the droplet height and coverage area of the droplet, as was also observed in our earlier work [31].

Dividing eq. (6) by (5), we obtain the color ratio (COR) of optical coupling in AM to FM as: 
(a)

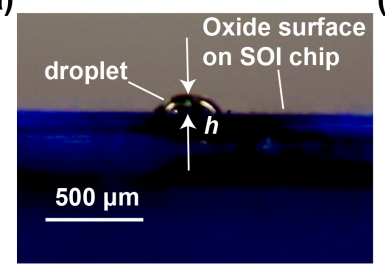

(b) 10

(c)

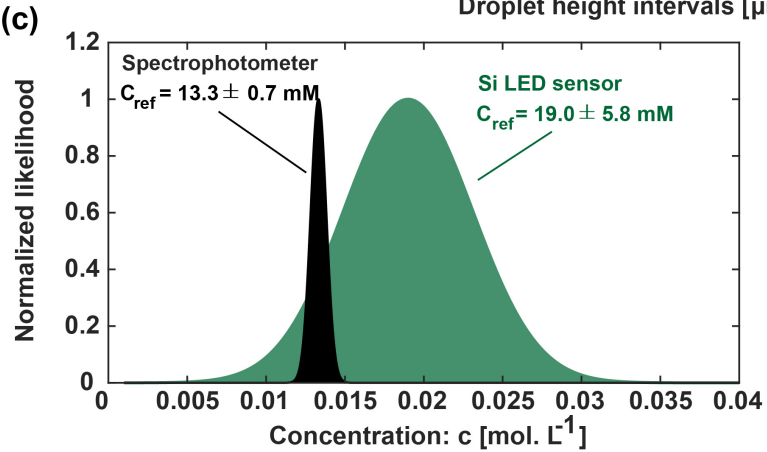

Fig. 7. (a) Optical micrograph of the side-view of a droplet-on-chip sampled from solution $\mathrm{C} 3$ showing the height profile. The estimated volume of the droplet was $\sim 0.1 \mu \mathrm{L}$. (b) Histogram showing the mean and standard deviation in the measured center-height of different droplets transferred to the chip with a silica fiber tip. (c) Estimated concentration of the target $\mathrm{NaCuChl}$ solution $\left(c_{\mathrm{ref}}\right)$ via the Si LED based sensor (green) and via the hybrid spectrophotometer (black). The standard error of the Gaussian curves represents the uncertainty in experiment, while the mean represents the estimated value.

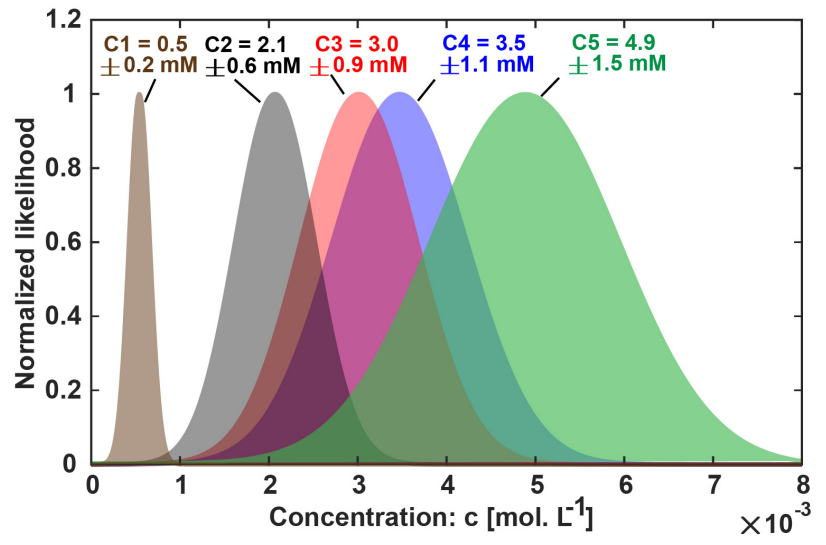

Fig. 8. Estimated concentrations of the diluted $\mathrm{NaCuChl}$ solution specimens $\mathrm{C} 1-\mathrm{C} 5$ via the Si LED based sensor, represented by a Gaussian likelihood curve. The standard error of the Gaussian curves represents the uncertainty in experiment, while the mean represents the estimated value.

$$
\begin{aligned}
\operatorname{COR}\left(c_{i}\right) & =\left(\frac{\Delta I_{\mathrm{PD}(\mathrm{Gly})}^{\mathrm{AM}}\left(c_{i}\right)}{\Delta I_{\mathrm{PD}(\mathrm{Gly})}^{\mathrm{FM}}\left(c_{i}\right)}\right) \\
& =\left(\frac{\Delta I_{\mathrm{PD}(\mathrm{Gly})}^{\mathrm{AM}}\left(c_{0}\right)}{\Delta I_{\mathrm{PD}(\mathrm{Gly})}^{\mathrm{FM}}\left(c_{0}\right)}\right) \cdot \exp \left[-\alpha\left(c_{i}\right) \cdot h\right] \\
& =\mathrm{COR}\left(c_{0}\right) \cdot \exp \left[-\alpha\left(c_{i}\right) \cdot h\right]
\end{aligned}
$$

where $\alpha\left(c_{i}\right)=\alpha_{\mathrm{M}}^{\mathrm{LED}} \cdot c_{i}$ is the effective absorption coefficient of the solution $\mathrm{C} i$ from which the droplet is sampled, and $c_{0}$ represents pure glycerol (hence, $\alpha=0$ ). Fig. 6 shows the measured COR versus relative concentration $c / c_{\text {ref }}$. The values are averaged over the set with $I_{\mathrm{LED}}=[1,2,3,4,5,6] \mathrm{mA}$. We observe that COR decreases with increasing $c$ in accordance with Beer-Lambert law $(\propto \exp [-\alpha \cdot h])$. An exponential fit (leastsquares method) yields the attenuation constant $\beta=1.495$. Thus, we obtain the concentration of the target solution $c_{\text {ref }}$ as

$$
c_{\mathrm{ref}}=\frac{\beta}{h \cdot \alpha_{\mathrm{M}}^{\mathrm{LED}}}
$$

The droplet height $h$ is estimated from optical microscopy as shown in Fig. 7(a). For droplets of size ranging from $\sim 100 \mu \mathrm{m}-\sim 1 \mathrm{~mm}, h$ is primarily governed by the angle of contact [40], [41] at the local liquid-chip $\left(\mathrm{SiO}_{2}\right)$ interface and the surface tension. Both parameters have uncertainties due to local surface properties and impurities (including the pigment) in the solvent. This results in a statistical variation in $h$, which is captured in Fig. 7(b) by analyzing multiple glycerol droplets on multiple dies. We observe $h=164.50 \pm 50.24 \mu \mathrm{m}$, where the error represents the standard deviation under a uni-modal Gaussian fit. Hence, using eq. (8) we obtain $c_{\text {ref }}=19.0 \pm 5.8$ mmol.L ${ }^{-1}(\mathrm{mM})$. Fig. $7(\mathrm{c})$ shows this result graphically with a Gaussian likelihood curve, where the error in our estimation is represented by the standard deviation $(\sigma)$. We validate the estimated $c_{\text {ref }}$, by an independent absorbance measurement on the specimen $\mathrm{C} 1$, using the hybrid spectrophotometer (SP) set-up as:

$$
c_{\mathrm{ref}}^{\mathrm{SP}}=\left(\frac{\alpha_{\mathrm{C} 1}(626 \mathrm{~nm})}{\alpha_{\mathrm{M}}(626 \mathrm{~nm})}\right) \cdot\left(\frac{c_{\mathrm{ref}}}{c_{1}}\right)
$$

where $\alpha_{\mathrm{C} 1}(626 \mathrm{~nm})$ is obtained from eq. (2). This gives $c_{\mathrm{ref}}{ }^{\mathrm{SP}}=13.3 \pm 0.7 \mathrm{mmol} . \mathrm{L}^{-1}(\mathrm{mM})$, which is in a close agreement with that obtained by our droplet sensing method with the broad-band Si LED. A small positive deviation in the estimated value with our sensor is likely due to an underestimation of $\alpha_{\mathrm{M}}^{\mathrm{LED}}$, where we have ignored the contribution of the weak absorption tail of $\mathrm{NaCuChl}$ for $\lambda>900 \mathrm{~nm}$ in eq. (4). Error could also arise due to possible inhomogeneities in the diluted specimens (high viscosity of glycerol).

For specimens $\mathrm{C} 1$ to $\mathrm{C} 5$, the concentrations can also be obtained independently using eq. (7) as

$$
c_{i}=\left(\frac{1}{h \cdot \alpha_{\mathrm{M}}^{\mathrm{LED}}}\right) \cdot\left(-\ln \left[\frac{\mathrm{COR}\left(c_{i}\right)}{\operatorname{COR}\left(c_{0}\right)}\right]\right)
$$

which yields $c_{1}=0.5 \pm 0.2 \mathrm{mM}, \quad c_{2}=2.1 \pm 0.6 \mathrm{mM}$, $c_{3}=3.0 \pm 0.9 \mathrm{mM}, c_{4}=3.5 \pm 1.1 \mathrm{mM}$, and $c_{5}=4.9 \pm 1.5 \mathrm{mM}$, as shown graphically in Fig. 8. Note that the standard error in the estimations propagates predominantly from the error in $h$. Taking the $1^{\text {st }}$ order derivative approximation of eq. (10), we get $\delta c_{i}=c_{i} \cdot(\delta h / h)$. Consequently, the absolute error is proportional to the concentration $c_{i}$. In order to evaluate the sensitivity $S_{\text {droplet }}$ of our sensor, we apply the differential approximation to eq. (7) that gives us

$$
S_{\text {droplet }}=\frac{\delta \mathrm{COR}\left(c_{i}\right)}{\delta c_{i}}=-\operatorname{COR}\left(c_{i}\right) \cdot \alpha_{\mathrm{M}}^{\mathrm{LED}} \cdot h
$$




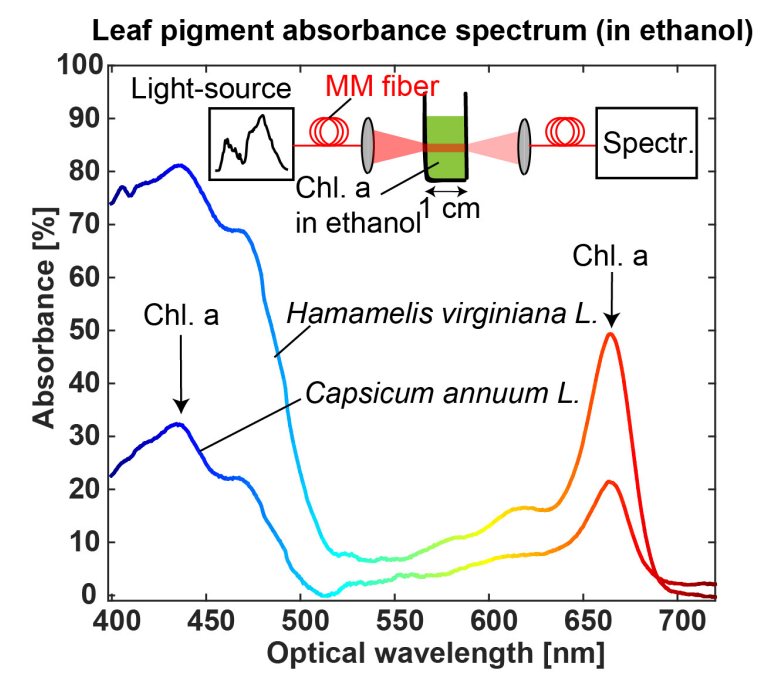

Fig. 9. Measured spectral absorbance of ethanolic solutions of photosynthetic pigments extracted from Hamamelis and Capsicum leaf samples, treated with $12 \mathrm{~mL}$ ethanol for 2 hours at $300 \mathrm{~K}$. The peak at $\boldsymbol{\lambda} \approx 660 \mathrm{~nm}$ is a signature of Chl. a, while the local peak at $\lambda \approx 475 \mathrm{~nm}$ indicates the presence of carotenoids. (Inset): Hybrid spectrophotometer set-up used for the absorbance measurement.

where the minus sign indicates that COR decreases with increasing $c$. At low concentrations, e.g. for specimen $\mathrm{C}$, we obtain $\left|S_{\text {droplet }}\right|=330 \mathrm{~mol}^{-1} \mathrm{~L}$.

\section{Non-invasive sensing of Chl. deficiency in leaves}

Chlorophyll (Chl. a,b) and carotenoids ( $\beta$-carotene) are the two naturally occurring dominant photosynthetic pigments [6], crucial for the growth and nutrition in plants. Reduction of Chl in leaves (chlorosis) is an important marker of plant stress. The measured absorbance $(A)$ spectra of pigments extracted (in ethanol) from leaves of two example species, namely Capsicum annuum L. and Hamamelis virginiana L., are shown in Fig. 9. The ethanolic extracts were sampled after a duration of 2 hours of dissolution at $300 \mathrm{~K}$, and analyzed with the hybrid spectrophotometer set-up (see inset). Both spectra exhibit peaks at $\lambda_{\mathrm{R}} \approx 660 \mathrm{~nm}, \lambda_{\mathrm{B}} \approx 475 \mathrm{~nm}$, and $\lambda_{\mathrm{V}} \approx 430 \mathrm{~nm}$. The peaks at $\lambda_{\mathrm{R}}$ and $\lambda_{\mathrm{V}}$ are attributed to Chl. $\mathrm{a}$, and the peak at $\lambda_{\mathrm{B}}$ is a signature of $\beta$-carotene [6], where small deviations $( \pm 5 \mathrm{~nm})$ [42] in peak positions may occur due to different solvents used. We observe that the AM EL spectrum of the Si LED has a maximum overlap with the absorption of Chl. a, followed by a relatively smaller overlap with that of carotene.

The COR was measured for the Capsicum and Hamamelis leaves at different timestamps of ethanol treatment. This was done by placing the leaves on top of the Si CMOS LED in our sensor and measuring light transmission through the leaf. The COR in the PD photocurrent against the measured absorbance of the corresponding ethanolic extract sampled immediately after withdrawing the Capsicum and Hamamelis leaf from the vial, are shown in Fig. 10(a) and (b), respectively. Also shown in the same figure are the corresponding Chl. a concentrations (c) (in solution) calculated from measured $A(660 \mathrm{~nm})$ and the literature-reported [43]-[45]

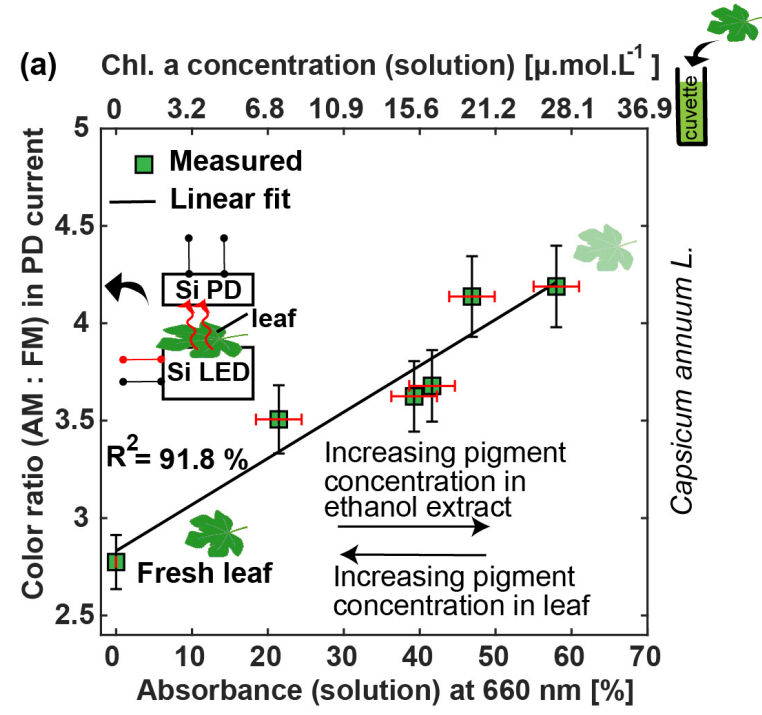

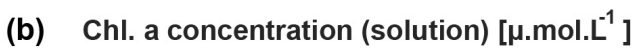

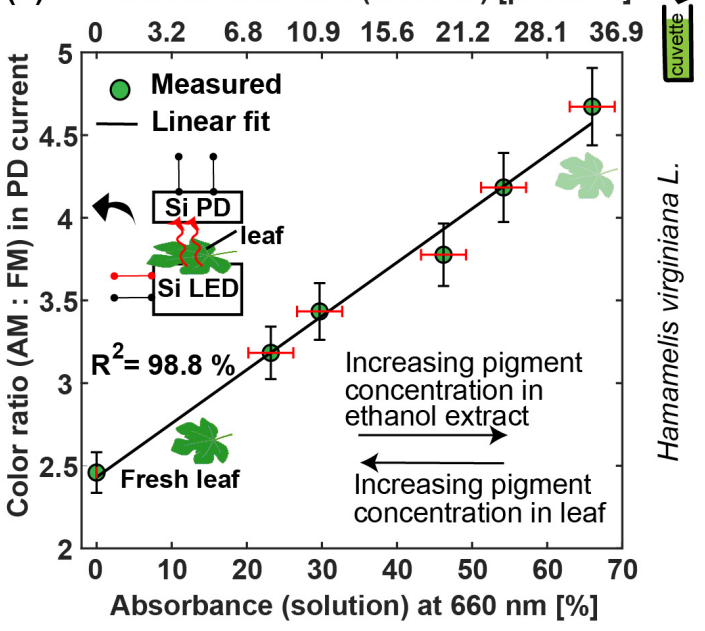

Fig. 10. COR (for the leaf) versus $660 \mathrm{~nm}$ absorbance (in ethanolic solution) for (a) Capsicum and (b) Hamamelis leaf samples with indicated error bars (symbols). The COR was measured by placing the leaf on the Si CMOS LED (see inset). The black solid line represents the linear fit, with a slope representing the mean sensitivity $(\delta \mathrm{COR} / \boldsymbol{\delta} \boldsymbol{A}[\%])$. The top $\mathrm{X}$-axis maps the absorbance to the equivalent $\mathrm{Chl}$. a concentration by using $\alpha_{\mathrm{M}(\mathrm{ChlA})}(660 \mathrm{~nm})=32640 \mathrm{~cm}^{-1} \mathrm{~mol}^{-1} \mathrm{~L}$.

$\alpha_{\mathrm{M}(\mathrm{ChlA})}(660 \mathrm{~nm})$ of $32640 \mathrm{~cm}^{-1} \mathrm{~mol}^{-1} \mathrm{~L}$. We observe a linear relationship (high correlation) between the COR and $A$. This is expected because the total amount of pigment molecules is conserved during the physical dissolution process. Hence, as the optical transmission through the leaf increases, the [Chl. a] in solution increases. The slope of the linear fit gives us $\delta \mathrm{COR} / \delta A[\%]=0.024$ and 0.0325 for Capsicum and Hamamelis respectively. Considering an error margin of \pm 0.2 in the COR (measured through the leaf), we obtain a resolution (and hence the detection limit) of absorbance $\delta A_{\text {res }}[\%]$ of $\approx 8.3 \%$ and $\approx 6.2 \%$ for the Capsicum and Hamamelis leaf respectively. Further, using a differential approximation of eq. (2), we can calculate the detection limit in terms of concentration (in solution) $c$ as: 


$$
\delta c_{\mathrm{res}}=\left(\frac{1}{\alpha_{\mathrm{M}(\mathrm{ChlA})} \cdot L_{\mathrm{opt}}}\right) \cdot\left(\frac{\delta A_{\mathrm{res}}}{100-A[\%]}\right)
$$

Thus, we obtain $\delta c_{\text {res }} \approx 5 \mu \mathrm{M}$ for Capsicum and $\approx 3.8 \mu \mathrm{M}$ for Hamamelis respectively, for a reference value of $A=50 \%$ (in solution), and $L_{\mathrm{opt}}=1 \mathrm{~cm}$ (path length in cuvette).

Further we can define the mean sensitivity $\left(S_{\text {leaf }}\right)$ of our sensor as

$$
\begin{array}{r}
S_{\text {leaf }}=\frac{\delta \mathrm{COR}}{\delta\left(c_{\mathrm{ChlA}}\right)}=\frac{\delta \mathrm{COR}}{\delta A[\%]} \cdot \frac{\delta A[\%]}{\delta\left(c_{\mathrm{ChlA}}\right)} \\
=\left(\frac{\delta \mathrm{COR}}{\delta A[\%]}\right) \cdot \alpha_{\mathrm{M}(\mathrm{ChlA})} \cdot L_{\mathrm{opt}} \cdot(100-A[\%])
\end{array}
$$

where $A$ is obtained by rearranging eq. (2) as $100 \times[1-$ $\left.\exp \left(-\alpha_{\mathrm{M}(\mathrm{ChlA})} \cdot L_{\mathrm{opt}} \cdot \Delta n_{\mathrm{ChlA}} / V_{\mathrm{sol}}\right)\right] . S$ depends on $\Delta n_{\mathrm{ChlA}}$. Substituting values, we obtain $S_{\text {leaf }}=3.9 \times 10^{4} \mathrm{~mol}^{-1} \mathrm{~L}$ and $5.3 \times 10^{4} \mathrm{~mol}^{-1} \mathrm{~L}$ for the Capsicum and the Hamamelis leaf, respectively, for a reference $A=50 \%$. Differences in $S$ between the two leaf species obtained by this method is due to sample-to-sample variation. This can be attributed to the different optical paths through the different cellular arrangements in the inner mesophyll tissue of the two leaves, and the different spatial distribution of Chl.

\section{DISCUSSION AND OUTLOOK}

We have presented the first proof-of-concept small-volume $(\sim 0.1 \mu \mathrm{L})$ optical absorption sensor with broad-spectrum avalanche-mode Si LEDs in standard CMOS technology. The same LED emits light with two disjoint and bias-dependent EL-spectra, only one of which is absorbed by a target specimen. This obviates the need for modifications in fabrication process and material composition, which are typically employed in tailoring the efficiency and EL-wavelength of Sibased light sources, as summarized in Table I. We outline the performance, future scope and applicability of our sensor under the following headings:

\section{A. Resolution and Sensitivity}

In solution phase, the dominant source of error in $c_{\text {ref }}$ in our experiment is the uncertainty in $h$, and thus, not limited by the LED or PD. For specimen C1 (the lowest concentration), we obtain a resolution of $\sim 200 \mu \mathrm{M}$ (Fig. 8). If the droplet height is known precisely, then the error in our experiment will be dominated by the $4 \%$ relative uncertainty in measuring $\operatorname{COR}\left(c_{i}\right)$. Using eq. (10), we then obtain that $\delta c_{1} / c_{1}$ to be $\sim 8 \%$. This amounts to an absolute error of $40 \mu \mathrm{M}$. Increasing the precision of the dispensed volume of the micro-droplet is envisaged in future developments, which will reduce the error and hence increase the resolution towards state-of-theart ( $\sim 1 \mu \mathrm{M})$ [46], [47] that uses hybrid quasi-monochromatic LEDs with III-V semiconductors in relatively bulky sensors. Eqs. (11) indicates that at a given reference concentration, sensitivity is proportional to the optical path length through which absorption takes place. In the non-invasive detection via a leaf, we emulate $\mathrm{Chl}$ stress by systematic reduction in the photosynthetic pigments via the ethanol treatment. The inner micro-structure of a leaf is largely a matrix of loosely packed mesophyll cells with a certain fraction of inter-cellular voids [48]. Light from the Si LED propagates through these mesophyll cells in a diffuse manner (multiple Fresnel reflections at the cell-void interfaces) [49], [50] before emerging into air and being collected by the photodiode. The light from the LED is divergent and hence it illuminates a patch of area $\sim 10 \mathrm{~cm}^{2}$ on the leaf. The color ratio measured is therefore averaged out over this spot, which relaxes the constraints on precise alignment. Thus, the net optical path is folded by a large leaf-dependent factor. Hence, detection with the intact leaf is expected to yield a higher sensitivity, as compared to the measurement in solution. The only trade-off in using a leaf directly is the inclusion of local variations in pigment distribution and optical path length through the leaf tissue in a single leaf.

\section{B. Stability}

Glycerol, being a low-voltatile (boiling points $>400 \mathrm{~K}$ ) [51], polar and organic solvent, is a suitable choice for the medium to dispense stable micro-droplets. The back-end oxide layer in the CMOS chip provides good passivation to shield the Si LED from chemicals in the droplet. Post-removal of droplet with iso-propanol, the chip surface was re-used for the next droplet with negligible change in $\Delta I_{\mathrm{PD}}$. However, only the Al-capped bond pads limit the degree of re-usability of the sensor in solution-phase due to possible (electro-)chemical side-reactions with and glycerol [52], [53] and water. For sensing carmine [31], the same device could be re-used for upto 6 droplet specimens, while for $\mathrm{NaCuChl}$ upto 3 droplets could be measured, before the Al-capping was corroded. For a given droplet, the measurements shown in Fig. 5 were stable for at least $\sim 5-7$ minutes. Longer duration of LED operation resulted in reactions between the Al-capped bond pads and glycerol, which in turn affects the shape of the droplet and pigment homogeneity.

\section{Device footprint and power consumption}

With the exception of the off-chip Si PD, our sensor has a footprint of less than $\sim 0.5 \mathrm{~mm}^{2}$, which includes a dualspectrum light-source within an area of $\sim 0.01 \mathrm{~mm}^{2}$. For industrial CMOS technologies, the production costs for silicon are typically $10-50$ eurocents per $\mathrm{mm}^{2}$ of die area. So, the cost of a single Si LED is estimated to be within a eurocent. Monolithic integration of the Si PD with a dedicated layout will further reduce the form factor of the sensor, which will be studied in our future work. From the viewpoint of fieldimplementation of such a sensor, either open-cavity or glasslid packaging would be preferred. In the latter scenario, an additional $\sim 7 \%$ loss in optical power is expected due to reflections at the air-glass interfaces. The dc power consumption in our LED ranged from $18 \mathrm{~mW}\left(I_{\mathrm{LED}}=1 \mathrm{~mA}\right)$ till $118 \mathrm{~mW}\left(I_{\mathrm{LED}}=6 \mathrm{~mA}\right)$. For each $I_{\mathrm{LED}}$, an integration time of $\sim 500 \mathrm{~ms}$ was used to reduce the noise in measuring the corresponding photocurrent with the SMU, leading to a 
TABLE I

PERFORMANCE COMPARISON OF SI-BASED LIGHT-EMITTING DEVICES IN LITERATURE

\begin{tabular}{|c||c||c||c||c||c|}
\hline $\begin{array}{c}\text { Light-emitter type } \\
\text { type }\end{array}$ & $\begin{array}{c}\text { Typical EL } \\
\text { wavelength [nm] }\end{array}$ & $\begin{array}{c}\text { Optical power efficiency } \\
\text { [order of magnitude] }\end{array}$ & $\begin{array}{c}\text { Standard CMOS } \\
\text { compatibility }\end{array}$ & $\begin{array}{c}\text { Wavelength } \\
\text { selection }\end{array}$ & References \\
\hline \hline $\begin{array}{c}\text { c-Si p-n junction } \\
\text { (this work) }\end{array}$ & $\begin{array}{c}400-900 \text { (avalanche) } \\
\sim 1100 \text { (forward) }\end{array}$ & $10^{-3}-10^{-6}$ & Yes & $\begin{array}{c}\text { By switching } \\
\text { bias polarity }\end{array}$ & $\begin{array}{c}{[19],[20],} \\
{[24],[26]}\end{array}$ \\
\hline $\begin{array}{c}\text { Si-on-insulator } \\
\text { FinFET technology }\end{array}$ & $\sim 1100$ & $10^{-3}$ & No & Fixed & {$[54]$} \\
\hline $\begin{array}{c}\text { Si nanocrystals } \\
\text { in SiO }\end{array}$ & $700-900$ & $10^{-3}$ & Yes & Fixed by \\
\hline $\begin{array}{c}\text { Strained Si-Ge } \\
\text { fabrication process }\end{array}$ & $\begin{array}{c}\text { [55], [56], } \\
{[57]}\end{array}$ & $\begin{array}{c}\text { Fixed by } \\
\text { stoichiometry }\end{array}$ & $\begin{array}{c}\text { [58], [59], } \\
{[60],[61]}\end{array}$ \\
\hline Er-doped Si & $1200-1600$ & $10^{-2}$ & No & Fixed by & {$[55],[62]$} \\
\hline
\end{tabular}

minimum energy consumption of $10 \mathrm{~mJ}$. In a prior work, the thermal resistance on the chip at a distance of $10 \mu \mathrm{m}$ from the LED was extracted to be $35 \mathrm{~K} / \mathrm{W}$ [27]. Thus, for an electrical input power of $20 \mathrm{~mW}$, the increase in steady-state temperature due to self-heating in the LED is within $1 \mathrm{~K}$. The CMOS technology is widely successful to provide high-level monolithic integration for electronic driver/read-out circuits. Pushing the limits of silicon based light sources, therefore, helps in miniaturizing such sensors and provide a low-cost alternative for coarse and rapid testing of food/water quality and plant health, where high precision is not critical.

\section{CONCLUSION}

We reported the first proof-of-principle of optical absorption sensing of pigment in solution with a broad-spectrum silicon micro LED designed in a standard silicon-on-insulator CMOS technology. Vertical optical transmission through a glycerol micro-droplet containing a chlorophyll based pigment (sodium copper chlorophyllin) was measured with a silicon photodiode. The on-chip silicon LED was driven in both forward and avalanche modes of operation, which steered its electroluminescent spectrum between visible and near-infrared, without replacing the droplet. The effective absorption coefficient and consequently the concentration in solution were determined from the color ratio (COR) of the measured photocurrent and the molar absorption coefficient of the pigment; the results were validated with a spectrophotometer. We validated our sensor further by employing it as a non-invasive chlorophyll sensor for stress monitor in plant leaves. Light emitted from the LED propagates through the leaf diffusely and undergoes absorption by the cellular chlorophyll before being detected by the photodiode. The COR of the photocurrent was shown to vary linearly with the decrease of chlorophyll from the leaf. Our work demonstrates the viability of a CMOS-integrated silicon LED, as a light source for micro-volume optical sensing.

\section{ACKNOWLEDGMENT}

The work has been carried out under the Plantenna research program funded by the 4TU Federation of the Netherlands.
The authors would like to thank NXP Semiconductors B.V. for silicon device fabrication. We thank Gideon Emmaneel, Rob Luttjeboer, and Xianfeng Chen from the department of Precision and Microsystems Engineering, TU Delft for technical support. We also thank Jurriaan Schmitz and Raymond J.E. Hueting from University of Twente for permitting to use the silicon devices.

\section{REFERENCES}

[1] H. Scheer," An Overview of Chlorophylls and Bacteriochlorophylls: Biochemistry, Biophysics, Functions and Applications, In: B. Grimm, R.J. Porra, W. Rüdiger, H. Scheer (eds.) Chlorophylls and Bacteriochlorophylls, Advances in Photosynthesis and Respiration, Springer, Dordrecht, vol. 25, chapter 1, pp. 1-26, 2006.

[2] A. Uchoa, A.M. Konopko, and M.S. Baptista, "Chlorophyllin derivatives as photosynthesizers: synthesis and photodynamic properties," J. Braz. Chem. Soc., vol. 26, no. 12, pp. 2615-2622, Dec. 2015.

[3] D. Cui, M. Li, and Q. Zhang, "Development of an optical sensor for crop leaf chlorophyll content detection," Computers and Electronics in Agriculture, vol. 69, no. 2, pp. 171-176, Dec. 2009.

[4] J.T. Alander, V. Bochko, B. Martinkauppi, S. Saranwong, and T. Mantere, "A review of optical nondestructuve visual and near-infrared methods for food quality and safety," International J. Spectroscopy, vol. 2013, 341402, Mar. 2013.

[5] L. Zeng, and D. Li, "Development of in situ sensors for chlorophyll concentration measurement," J. Sensors, vol. 2015, 903509, Apr. 2015.

[6] H. Croft, J.M. Chen, "Leaf Pigment Content," Comprehensive Remote Sensing, Shunlin Lian (ed.), Elsevier, Oxford, vol. 3, chapter 9, pp. 117142, 2018.

[7] S. Bergsträsser, D. Fanourakis, S. Schmittgen, M.P. Cendrero-Mateo, M. Jansen, H. Scharr and U. Rascher, "HyperART: non-invasive quantification of leaf traits using hyperspectral absorption-reflectancetransmittance imaging," Plant Methods, vol. 11, no. 1, Jan. 2015.

[8] H. Gao, C. Yan, W. Wu and J. Li, "Application of microfluidic chip technology in food safety sensing," Sensors, vol. 20(6): 1792, Mar. 2020.

[9] M.O' Toole and D. Diamond, "Absorbance based light emitting diode optical sensors and sensing devices," Sensors, vol. 8, pp. 2453-2479, Apr. 2008.

[10] F.M. Padilla, R. de Souza, M.T. Peña-Fleitas, M. Gallardo, C. Giménez, and R.B. Thompson, "Different responses of various chlorophyll meters to increasing nitrogen supply in sweet pepper," Front. Plant Sci., vol. 9, 1752, Nov. 2018

[11] G. Minas, J.C. Ribeiro, R.F. Wolffenbuttel, and J.H. Correia, "On-chip integrated CMOS optical detection microsystem for spectrophotometric analyses in biological microfluidic systems," proceedings IEEE International Symposium of Industrial Electronics (ISIE)., pp.1133-1138, Jun. 2005.

[12] R. Soref, "Applications of silicon-based optoelectronics," MRS Bulletin, vol. 23, no. 4, pp. 20-24, Apr. 1998. 
[13] L.W. Snyman, H. Aharoni, A. Biber, A. Bogalecki, L. Canning, M. du Plessis, and P. Maree, "Optical sources, integrated optical detectors, and optical waveguides in standard silicon CMOS integrated circuitry," proceedings SPIE, vol. 3953, Silicon-based Optoelectronics II, pp. 20-36, Mar. 2000.

[14] U. Lu, B.C.-P. Hu, Y. -C Shih, Y. -S Yang, C. -Y. Wu, C. -J. Yuan, M. -D. Ker, T. -K. Wu, Y. -K. Li, Y. -Z. Hsieh, W. Hsu, and C. -T. Lin, "CMOS chip as luminescent sensor for biochemical reactions," IEEE Sensors J., vol. 3, no. 3, pp. 310-316, Jun. 2003.

[15] F. J. Blanco, M. Agirregabiria, J. Berganzo, K. Mayora, J. Elizalde, A. Calle, C. Domniguez, and L.M. Lechuga, "Microfluidic-optical integrated CMOS compatible devices for label-free biochemical sensing," J. Micromech. Microeng., vol. 16, pp. 1006-1016, Apr. 2006.

[16] K. De Vos, I. Bartolozzi, E. Schacht, P. Bienstman, and R. baets, "Silicon-on-insulator microring resonator for sensitive and label-free biosensing," Opt. Express, vol. 15, no. 12, pp. 7610-7615, Jun. 2007.

[17] A. Frey, M. Schienle, and H. Seidel, "CMOS based sensors for biochemical analysis," proceedings IEEE Transducers, pp. 1670-1673, Jun. 2009.

[18] C. Sun et al., "Single-chip microprocessor that communicates directly using light," Nature, vol. 528, pp. 534-538, Dec. 2015.

[19] K. Xu, Y. Chen, T.A. Okhai, and L.W. Snyman, "Micro optical sensors based on avalanching silicon light-emitting devices monolithically integrated on chips," Opt. Mat. Express, vol. 9, no. 10, pp. 3985-3997, Oct. 2019.

[20] M. Green, J. Zhao, A. Wang, P.J. Reece, and M. Gal, "Efficient silicon light emitting diodes," Nature, vol. 412, no. 6849, pp. 805-808, Aug. 2001.

[21] T. Trupke, J. Zhao, A. Wang, R. Corkish, and M. Green, "Very efficient light emission from bulk crystalline silicon," Appl. Phys. Lett., vol. 82, no. 18, pp. 2996-2998, Apr. 2003.

[22] A.G. Chynoweth and G. McKay, "Photon emission from avalanche breakdown in silicon," Phys. Rev., vol. 102, no. 2, pp. 369-376, Apr. 1956.

[23] S. Dutta, R.J.E. Hueting, A.-J. Annema, L. Qi, L.K. Nanver, and J. Schmitz, "Opto-electronic modeling of light emission from avalanchemode silicon $\mathrm{p}^{+} \mathrm{n}$ junctions," J. Appl. Phys., vol. 118, 114506, Sep. 2015.

[24] L.W. Snyman, M. du Plessis, and H. Aharoni, "Injection-avalanchebased $\mathrm{n}^{+}$pn silicon complementary metal-oxide-semiconductor lightemitting device (450-750 nm) with 2-order-of-magnitude increase in light emission intensity,' Jpn. J. Appl. Phys., vol. 46, no. 4B, pp. 2474 2480, Apr. 2007.

[25] L.W. Snyman, and K. Xu, "Stimulation of 450, 650, and $850 \mathrm{~nm}$ optical emissions from custom designed silicon LED devices by utilizing carrier energy and carrier momentum engineering," proceedings SPIE, vol. 10036, 1003603, Feb. 2017

[26] S. Dutta, G.J.M. Wienk, R.J.E. Hueting, J. Schmitz, and A.-J. Annema, "Optical power efficiency versus breakdown voltage of avalanche-mode silicon LEDs in CMOS,'IEEE Electron Device Lett., vol. 38, no. 7, pp.898-901, Jul. 2017.

[27] S. Dutta, V. Agarwal, R.J.E. Hueting, J. Schmitz, and A.-J. Annema, "Monolithic optical link in silicon-on-insulator CMOS technology," Opt. Express, vol. 25, no. 5, pp. 5440-5456, Mar. 2017.

[28] B. Huang, X. Zhang, W. Wang, Z. Dong, N. Guan, Z. Zhang, and H. Chen, "CMOS monolithic optoelectronic integrated circuit for onchip optical interconnection," Opt. Comm., vol. 284, pp. 3924-3927, Aug. 2011.

[29] V. Agarwal, S. Dutta, A.-J. Annema, R.J.E. Hueting,J. Schmitz, M.J. Lee, E. Charbon, and B. Nauta, "Optocoupling in CMOS," proceedings IEEE $64^{\text {th }}$ International Electron Devices Meeting (IEDM), pp. 739-742, Dec. 2018.

[30] I. Gorczyca, T. Suski, N.E. Christensen, and A. Svane, "Limitations to band gap tuning in nitride semiconductor alloys," Appl. Phys. Lett., vol. 96, 101907, Mar. 2010.

[31] S. Dutta, P.G. Steeneken, and G.J. Verbiest, "Optical absorption sensing with dual-spectrum silicon LEDs in SOI-CMOS technology," proceedings IEEE Sensors 2020, p. 6498, Oct. 2020.

[32] P. Wessels, M. Swanenberg, H. van Zwol, B. Krabbenborg, H. Boezen, M. Berkhout, and A. Grakist, "Advanced BCD technology for automotive, audio and power applications," Solid-State Electronics, vol. 51, pp. 195-211, Feb. 2007.

[33] E.R. Hunt Jr., B.N. Rock, P.S. Rock, "Measuring of leaf water content by infrared reflectance," Remote Sensing of Environment, vol. 22, no. 3, pp. 429-435, Aug. 1987.

[34] H.W. Gausman, W.A. Allen, and D.E. Escobar, "Refractive index of plant cell walls," Appl. Opt., vol. 13, no. 1, pp. 109-111, Jan. 1974.
[35] A. Bricaud, A. Morel, and L. Prieur, "Absorption by dissolved organic matter of the sea (yellow substance) in the UV and visible domains," Limnology and Oceanography, vol. 26, no. 1, pp. 43-53, Jan. 1981.

[36] G. Pérez, C. Queimaliños, E. Balseiro, and B. Modenutti, "Phytoplankton absorption spectra along the water column in deep North Patagonian Andean lakes (Argentina)," Limnologica, vol. 37, no. 1, pp. 3-16, Feb. 2007.

[37] Z.P. Lee and K.I. Carder, "Absorption spectrum of phytoplankton pigments derived from hyperspectral remote-sensing reflectance," Remote Sensing of Environment, vol. 89, no. 3, pp. 361-368, Feb. 2004.

[38] M. Nitzan, A. Romem, and R. Koppel, "Pulse oximetry: fundamentals and technology," Medical devices: evidences and research, vol. 2014, no. 7, pp. 231-239, Jul. 2014.

[39] H. Kanemaru, S. Yukita, H. Namiki, Y. Nosaka, T. Kobayashi, and E. Tokunaga, "Giant Pockels effect of polar organic solvents and water in the electrical double layer on a transparent electrode," RSC Adv., vol. 7, no. 72, pp. 45682-45690, Sep. 2017.

[40] A. Moldovan, M. Bota, D. Dorobantu, I. Boerasu, D. Bojin, D. Buzatu, and M. Enachescu, "Wetting properties of glycerol on silicon, native $\mathrm{SiO} 2$, and bulk $\mathrm{SiO} 2$ by scanning polarization force microscopy," $J$. Adhesion Sci. and Tech., vol. 28, no. 13, pp. 1277-1287, Mar. 2014.

[41] F. Behroozi and P.S. Behroozi, "Reliable detection of contact angle from the height and volume of sessile drops," Am. J. Phys., vol. 87, no. 1, pp. 28-32, Jan. 2019.

[42] G.R. Seely and R.G. Jensen, "Effect of solvent on the spectrum of chlorophyll," Spectrochimica Acta, vol. 21, no. 10, pp. 1835-1845, Oct. 1965.

[43] H.H. Strain, M.R. Thomas, and J.J. Katz, "Spectral absorption properties of ordinary and fully deuteriated chlorophylls a and b," Biochimoca et Biophysica Acta, vol. 75, pp. 306-311, May 1963

[44] H.K. Lichtenthaler, "Chlorophylls and carotenoids: Pigments of photosynthetic biomembranes," In: L. Packer , R. Douce (eds.) Methods in Enzymology, Academic Press, vol. 148 (Plant Cell Membranes), chapter 34, pp. 350-382, 1987.

[45] H. Du, R.-C.A. Fuh, J. Li, L.A. Corkan and J.S. Lindsey, "PhotochemCAD: A computer-aided design and research tool in photochemistry," Photochem. Photobiol., vol. 68, no. 2, pp. 141-142, Aug. 1998.

[46] M.O' Toole, K.T. Lau, R. Shepherd, C. Slater and D. Diamond, "Determination of phosphate using highly sensitive paired emitter-detector diode photometric flow detector," Analytica Chimica Acta, vol. 597, pp. 290-294, Jun. 2007.

[47] J. Huang, H. Liu, A. Tan, J. Xu and X. Zhao, "A dual-wavelength lightemitting diode based detector for flow-injection analysis for process analysers," Talanta, vol. 39, no. 6, pp. 589-592, Jun. 1992.

[48] H.W. Gausman, W.A. Allen, R. Cardenas, and A.J. Richardson, "Relation of light reflectance to histological and physical evaluations of cotton leaf maturity," Appl. Opt., vol. 9, no. 3, pp. 545-552, Mar. 1970.

[49] E.A. Walter-Shea and J.M. Norman, "Leaf optical properties," In: R.B. Myneni , J. Ross (eds.) Photon-Vegetation Interactions, Springer, Berlin, Heidelberg, chapter 8, pp. 229-251, 1991.

[50] J.T. Woolley, "Reflectance and transmittance of light by leaves," Plant Physiology, vol. 47, no. 5, pp. 656-662, May 1971.

[51] L.B. Lane, "Freezing points of glycerol and its aqueous solutions," Ind. Eng. Chem., vol. 17, no. 9, pp. 924, Sep. 1925.

[52] H.B. Zhang, J.H. Yang, F. Gao, and J.J. Lin, "Experimental study of the breakdown characteristic of glycerol as energy storage medium in pulse forming line," proceedings Annual Report Conference on Electrical Insulation and Dielectric Phenomena, pp. 850-853, Oct. 2013.

[53] S. Schünemann, F. Schüth, and H. Tüysüz, "Selective glycerol oxidation over ordered mesoporous copper aluminium oxide catalysts," Catal. Sci. Technol., vol. 7, no. 23, pp. 5614-5624, Oct. 2017.

[54] G. Piccolo, P.I. Kuindersma, R.J.E. Hueting, N. Collaert, and J. Schmitz, "Silicon LEDs in FinFET technology," proceedings of the $44^{\text {th }}$ European Device Research Conference (ESSDERC), pp. 274-277, Sep. 2014.

[55] L. Pavesi, "Silicon-based light sources for silicon integrated circuits," Advances in optical technologies, vol. 2008, Article ID 416926, Jun. 2008.

[56] L. Pavesi, L. Dal Negro, C. Mazzoleni, G. Franzò, and F. Priolo, “Optical gain in silicon nanocrystals," Nature, vol. 408, pp. 440-444, Nov. 2000.

[57] R.J. Walters, G.I. Bourianoff, and H.A. Atwater, "Field-effect electroluminescence in silicon nanocrystals," Nature Materials, vol. 4, no. 2, pp. 143-146, Jan. 2005.

[58] J. Liu, X. Sun, D. Pan, X. Wang, L.C. Kimerling, T.L. Koch, and J. Michel, "Tensile-strained, n-type Ge as a gain medium for monolithic laser integration on Si," Opt. Express, vol. 15, no. 18, pp. 11272-11277, Aug. 2007. 
[59] B. Schwartz, A. Klossek, M. Kittler, M. Oehme, E. Kasper, and J. Schulze, "Electroluminescence of germanium LEDs on silicon: influence of antimony doping," Phys. Status Solidi C, vol. 11, no. 11-12, pp. 1686-1691, Oct. 2014.

[60] M. Kaschel, M. Schmid, M. Gollhofer, J. Werner, M. Oehme, and J. Schulze, "Room-temperature electroluminescence from tensile strained double-heterojunction germanium pin LEDs on silicon substrates," Solid-State Electronics, vol. 83, no. 11-12, pp. 87-91, May 2013.

[61] H. Li, S. Zhang, Z. Zhang, S. Zuo, S. Zhang, Y. Sun, D. Zhao, and Z. Zhang, "Silicon waveguide integrated with germanium photodetector for a photonic-integrated FBG interrogator," Nanomaterials, vol. 10, 1683, Aug. 2020.

[62] N. Daldosso, D. Navarro-Urrios, M. Melchiorri, C. Garca, P. Pellegrino, B. Garrido, C. Sada, G. Battaglin, F. Gourbilleau, R. Rizk, and L. Pavesi, "Er-coupled Si nanocluster waveguide," IEEE Journal on Selected Topics in Quantum Electronics, vol. 12, no. 6, pp. 1607-1617, Nov.-Dec. 2006. 